\title{
Nuevo método para la cuantificación de la actividad endoglucanasa basado en el complejo celulosa-rojo congo
}

\section{A new method for quantifying endoglucanase activity based on Congo red-carboxymethyl cellulosecomplex depolymerisation}

\author{
Martha L. Ortiz Moreno1 ${ }^{1}$ Daniel Uribe - Vélez² \\ ${ }^{1}$ Facultad de Ciencias Básicas e Ingeniería, Universidad de los Llanos \\ ${ }^{2}$ Instituto de Biotecnología, Universidad Nacional de Colombia \\ E-mail:mlortizm@unillanos.edu.co
}

Recibido: Agosto 3 de 2010. Aceptado: Octubre 5 de 2010

\begin{abstract}
RESUMEN
El objetivo de este trabajo fue establecer un método de cuantificación para la actividad endoglucanasa basado en la despolimerización del complejo polisacárido-rojo Congo, con este fin se describió el comportamiento matemático de la viscosidad y la absorbancia del medio de cultivo carboximetilcelulosarojo Congo. Para medir el impacto de un hongo celulolítico como Trichoderma viride sobre el medio, se realizó el seguimiento de la viscosidad y la absorbancia del medio de cultivo inoculado por 8 días. El cálculo de la concentración de enzima se realizó correlacionando los resultados de pruebas de azúcares reductores por DNS con la pérdida de absorbancia del medio de cultivo CMC. Para evaluar la aplicabilidad de la técnica se probaron cuatro cepas de deuteromicetos celulolíticos y se encontró que el uso de la variable pérdida de absorbancia del medio CMC, fue efectiva para diferenciar la actividad endoglucanasa de las cepas. EI método basado en rojo Congo-CMC es más rápido y sensible que el DNS. Se recomienda aplicar este nuevo método en los screening para la bioprospección de hongos celulolíticos.
\end{abstract}

Palabras clave: Endoglucanasa, carboximetilcelulosa, rojo Congo, hongos celuloliticos.

\begin{abstract}
The aim of this work was to establish a method for quantifying endoglucanase activity based on the depolymerisation of the Congo red-polysaccharide complex. The mathematical viscosity and absorbance patternsforCongo red-carboxymethylcellulose $(\mathrm{CMC})$ culture media were thus described. The inoculated media'sviscosity and absorbance were monitored for 8 daysto measure the impact of a cellulolytic fungus such as Trichodermaviride on the media. Enzymeconcentration was calculated by correlating reducing sugars'absorbance test results using3,5-dinitrosalicylic acid (DNS) with loss of CMC culture mediaabsorbance. Four cellulolytic deuteromycetesstrains were testedto assess the technique'sapplicability; it was found that usingCMC media absorbancelosswas effective for differentiatingstrains'endoglucanase activity. The Congored-
\end{abstract}


CMC method was faster and more sensitive than the DNS method. This new method should thusbe appliedin screening assays when bioprospecting cellulolytic fungi in soil.

Key words: Endoglucanase, carboxymethylcellulose, Congo red, cellulolytic fungus.

\section{INTRODUCCIÓN}

La bioprospección de microorganismos, enzimas y genes implicados en la degradación de la celulosa sigue estando dentro de los temas de investigación de punta en la microbiología aplicada y la biotecnología. La industria y la biorremediación exigen cada vez nuevos y más eficientes sistemas enzimáticos con capacidad de actuar en condiciones ambientales extremas y con altos rendimientos (Ohmiya et al. 2003; Aro et al. 2005).

Para lograr realizar una bioprospección eficiente se requieren metodologías rápidas, sensibles, replicables y económicas para comparar un alto número de aislamientos de microorganismos celulolíticos.

Cuantificar la actividad celulolítica representa un reto para la enzimología, debido a la complejidad del sistema enzimático implicado y a la heterogeneidad de las técnicas y unidades empleadas para reportarla por diferentes laboratorios. Con el fin de estandarizar los métodos de medición de la actividad celulolítica la IUPAC en 1984 publico un listado de técnicas de referencia, sin embargo no todas tuvieron gran acogida entre los biotecnólogos, ya que eran restrictivas en el uso de sustratos, dispendiosas o poco replicables (IUPAC 1984; Wood \& Bhat 1988; AOAC 2000).

Uno de los componentes más importante del sistema enzimático celulolítico es la enzima endo1,4- $\beta$ glucanasa, la cual hidroliza al interior de las cadenas de celulosa aleatoriamente. Esta enzima se encuentra en todos los organismos celulolíticos desde bacterias hasta hongos (Aro et al. 2005). Los sustratos en que se ha evaluado son: carboximetilcelulosa (CMC), celulosa pretratada con álcali o ácido y celulosa cristalina (fibra de algodón o avicel). Sin embargo sólo los sustratos de estructura amorfa pueden ser ampliamente despolimerizados por la endoglucanasa. Los productos de la actividad endoglucanasa son glucosa y celobiosa. Para cuantificar los productos enzimáticos se han empleado técnicas como: la detección de azúcares reductores por las técnicas de Somoyi-Nelson

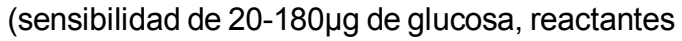
tóxicos), del ácido Dinitrosalicílico (20-180mg de glucosa) y Rojo Fenol (sensibilidad entre 20-180 $\mu \mathrm{g}$ de glucosa) y HPLC; disminución de la viscosidad (unidad arbitraria) o turbidez del sustrato (baja replicabilidad) e hidratación del algodón en álcali (unidad arbitraria) (Wood \& Bhat 1988; González \& Castellanos, 2003).

Otros métodos reportados para la actividad endoglucanasa son: el uso de celulosa en polvo ligada a un colorante reportero de la despolimerización (Smith, 1977). El uso de columnas opacas de medio sólido que contienen celulosa parcialmente cristalina, en el que se detecta la actividad celulolítica de hongos (Rautela \& Cowling 1966). La técnica cualitativa más usada es la reportada por Teather \& Wood (1982), que se basa en la asociación de rojo Congo y carboximetilcelulosa que genera una intensa coloración que se desvanece ante la actividad despolimerizante de la endoglucanasa. EI rojo Congo tiene una alta afinidad por los polisacáridos y ha sido utilizado para el diagnóstico de la amiloidosis (Bély et al. 2006), diferenciación de cepas patógenas de Escherichia coli (Berkhoff \& Vinal 1986), para el aislamiento de Azospirillum (Hernández et al. 2000), como colorante vital para raíces (Pire 1985) y para la descripción de tejidos fúngicos (Guzmán et al. 1999).

De todos los métodos anteriormente descritos el más empleado es del ácido Dinitrosalícilico (DNS) por su bajo costo y baja toxicidad de reactantes (Sengupta et al. 2000; Srinivasan et al. 2001; Hong 
et al. 2001; Nomachi et al. 2002; Chhabra \& Kelly 2002; Contreras-Pinzón et al. 2007), sin embargo, su insensibilidad a bajas concentraciones de glucosa y los grandes volúmenes de muestra requeridos (superiores a $5 \mathrm{ml}$ ), sugieren que es necesario

\section{MATERIALES Y MÉTODOS}

\section{Características del medio de cultivo CMC}

Para describir la viscosidad y absorbancia del medio de cultivo, se preparo soluciones del medio (Thorn et al, 1996 modificado en la fuente de carbono) con diferentes concentraciones de CMC y Rojo Congo al 0,029 \%. Las concentraciones empleadas fueron: $0,2 \% ; 0,4 \% ; 0,6 \% ; 0,8 \%$; $1,0 \%$. Se evaluó la viscosidad en centiPoissons/ segundo con un viscosímetro de cilindros de rotación coaxial (Haake, Rotovisco RV20) y se realizó la lectura de la absorbancia a 200 $\mu$ de muestra, en un lector de microplacas (Merck, Sensident Scan) a 488nm. Como control se empleo el medio de cultivo sin CMC. Este ensayo se realizó con tres réplicas y dos veces en el tiempo.

\section{Relación absorbancia-viscosidad del medio de cultivo bajo la acción de un hongo}

Con el fin de describir el comportamiento de la viscosidad y la absorbancia del medio de cultivo se realizaron pruebas con el hongo comercial Trichoderma viride, cultivándolo en el medio CMCrojo Congo $(100 \mathrm{ml})$ realizando lecturas de las variables diariamente. Se empleó una concentración de $\mathrm{CMC}$ del $0,8 \%$, debido a que era la máxima que permitía una solubilización rápida en la solución de sales. Las condiciones de incubación fueron $30^{\circ} \mathrm{C}$, agitación a $150 \mathrm{rpm}$ y oscuridad. El inóculo fue diez cubos de $1 \mathrm{~cm}^{3}$ de micelio vegetativo de $T$. viride crecido en agar CMC por 8 días. Las pruebas se realizaron por triplicado y repetidos dos veces en el tiempo.

\section{Evaluación de cepas problema}

Bajo las condiciones experimentales establecidas con $T$. viride, se realizó una prueba para comparar la actividad endoglucanasa entre varios desarrollar técnicas alternativas para el screening de alto número de aislamientos. El objetivo de este trabajo fue evaluar un nuevo método de cuantificación de la actividad endoglucanasa empleando el principio cualitativo reportado por Teather \& Wood (1982).

aislamientos fúngicos celulolíticos obtenidos de suelos con diferentes usos agrícolas de Puerto López (Meta). Se empleó la cepa 055C Penicillium spp; la cepa 065C Actinomucor spp, ambas aisladas de sabana y la cepa 019C Trichoderma spp obtenida de bosque, como cepa de referencia se utilizó a $T$. viride. Con tres réplicas por cepa y dos repeticiones en el tiempo.

\section{Relación absorbancia-unidades de enzima}

Prueba de DNS: En tres tubos de ensayo por cepa, con $10 \mathrm{ml}$ de medio CMC, se inocula 1 cubo de $1 \mathrm{~cm}^{3}$ por mililitro. Luego se incubaron a $25^{\circ} \mathrm{C}$ con 150 rpm de agitación por 15 días, se recuperó $1 \mathrm{ml}$ de sobrenadante de cada tubo y se centrifugó a 10000rpm por 10 minutos (centrifuga Eppendorf 5415C), luego se tomó 500 $\mu$ l de sobrenadante y se dispuso en un tubo eppendorf que contenía $500 \mu \mathrm{\mu l}$ de una solución de CMC 0,8 \% en buffer citrato de sodio $50 \mathrm{mM} \mathrm{pH}=4$ y se llevo a incubación a $50^{\circ} \mathrm{C}$ por 72 horas, ya que se desea evaluar la cantidad de glucosa producida por las endoglucanasas a partir de CMC. Las endoglucanasas son generadas por el hongo en la primera incubación. En la segunda incubación no hay biomasa fúngica para evitar que incorpore la glucosa generada (Brasil et al. 2004).

Finalmente a una muestra de cada tubo eppendorf se le estimó la concentración de glucosa producida por la acción de la enzima endoglucanasa por DNS de la siguiente manera: adicionar en un tubo de vidrio $150 \mu \mathrm{l}$ de la muestra a evaluar, agregar $350 \mu \mathrm{l}$ de agua miliQ y $500 \mu$ l de una solución que contiene DNS $(1 \% \mathrm{p} / \mathrm{v})$, hidróxido de sodio $(1,6 \% \mathrm{p} / \mathrm{v})$ y tartrato de sodio y potasio ( $30 \% \mathrm{p} / \mathrm{v})$, llevar los tubos a ebullición en un baño de maría por 5 minutos, retirar y enfriar en agua hielo. Posteriormente adicionar $4 \mathrm{ml}$ de agua miliQ, homogenizar por 
inversión 5 veces y dejar en reposo por 15 minutos. A continuación se toman $200 \mu l$ de la reacción y se depositan en una placa multipozo (96 pozos) para realizar la lectura a $541 \mathrm{~nm}$ en lector de microplacas (Sensident Scan, Merck). Las unidades de enzima se expresan en micromoles de enzima/minL.

\section{RESULTADOS}

\section{Descripción de las características del medio de cultivo CMC}

El medio de cultivo para la medición de la actividad endoglucanasa fue evaluado para describir su viscosidad y absorbancia. El comportamiento del medio de cultivo para las dos variables fue exponencial, con coeficientes de determinación superiores a 0,900 (Fig 1). Esto indica que la perdida de viscosidad no tiene un comportamiento lineal. El
El análisis de los datos se realizó con el paquete estadístico Minitab 14, aplicando análisis de regresión, análisis de varianza (ANOVA) y comparaciones múltiples. análisis de correlación mostró relación entre las variables, representada mediante la siguiente ecuación: Absorbancia $=0,0157+0,000167$ Viscosidad - 0,0260 \%CMC; con un coeficiente de determinación del 0,996. Lo cual indica que se puede predecir la absorbancia del medio en función de su viscosidad y concentración de CMC.

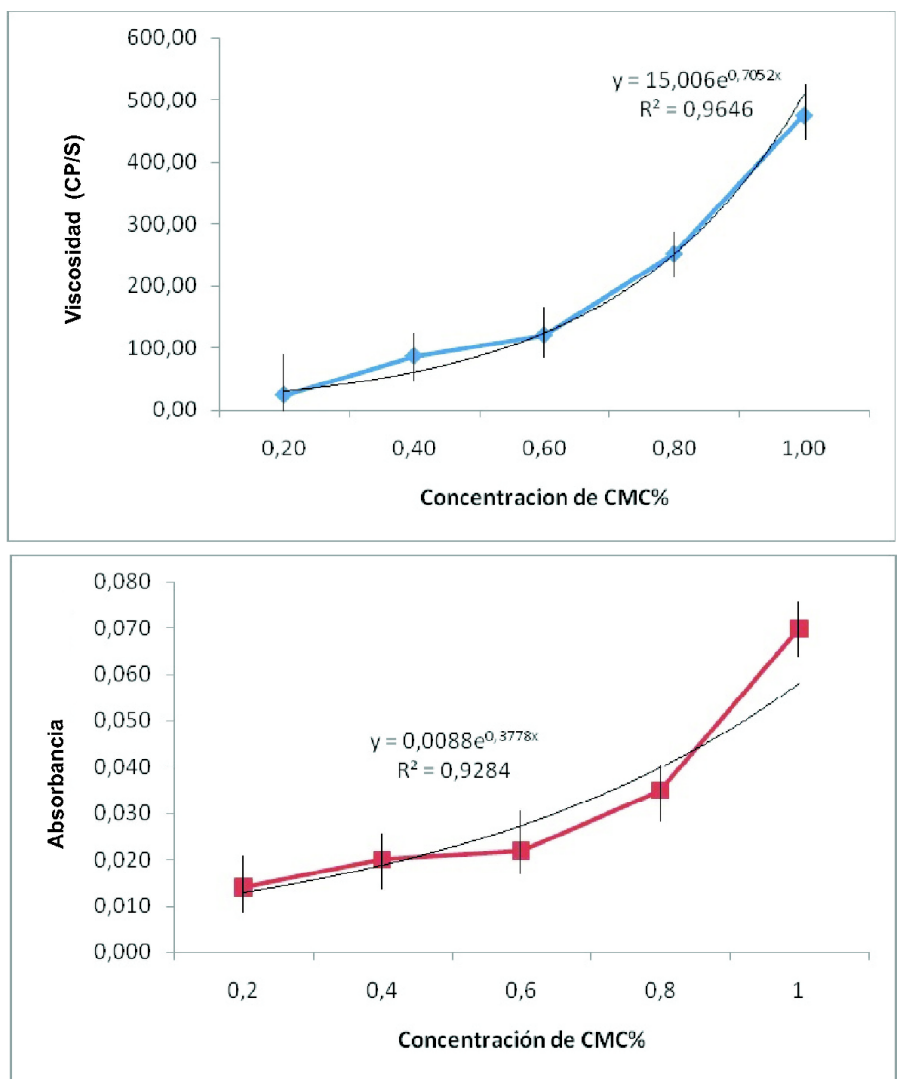

Figura 1. Comportamiento del medio de cultivo a medida que varia la concentración de CMC, A) Viscosidad; B) Absorbancia 
Determinación de la correlación entre unidades enzimáticas

Al exponer el medio de cultivo al hongo T. viride, se encontró que el hongo indujo una pérdida de viscosidad drástica del $98,2 \%$ en 5 días, a una tasa media del $19,7 \%$ diario. En el primer día el medio perdió el $78 \%$ de su viscosidad, en el segundo $8,5 \%$, en el tercero $7,7 \%$ y finalmente en el cuarto $4 \%$. Del quinto día al octavo, no hubo variaciones tanto en la viscosidad como en la absorbancia, tal y como lo indicaban las observaciones de los erlenmeyers (Fig 2).

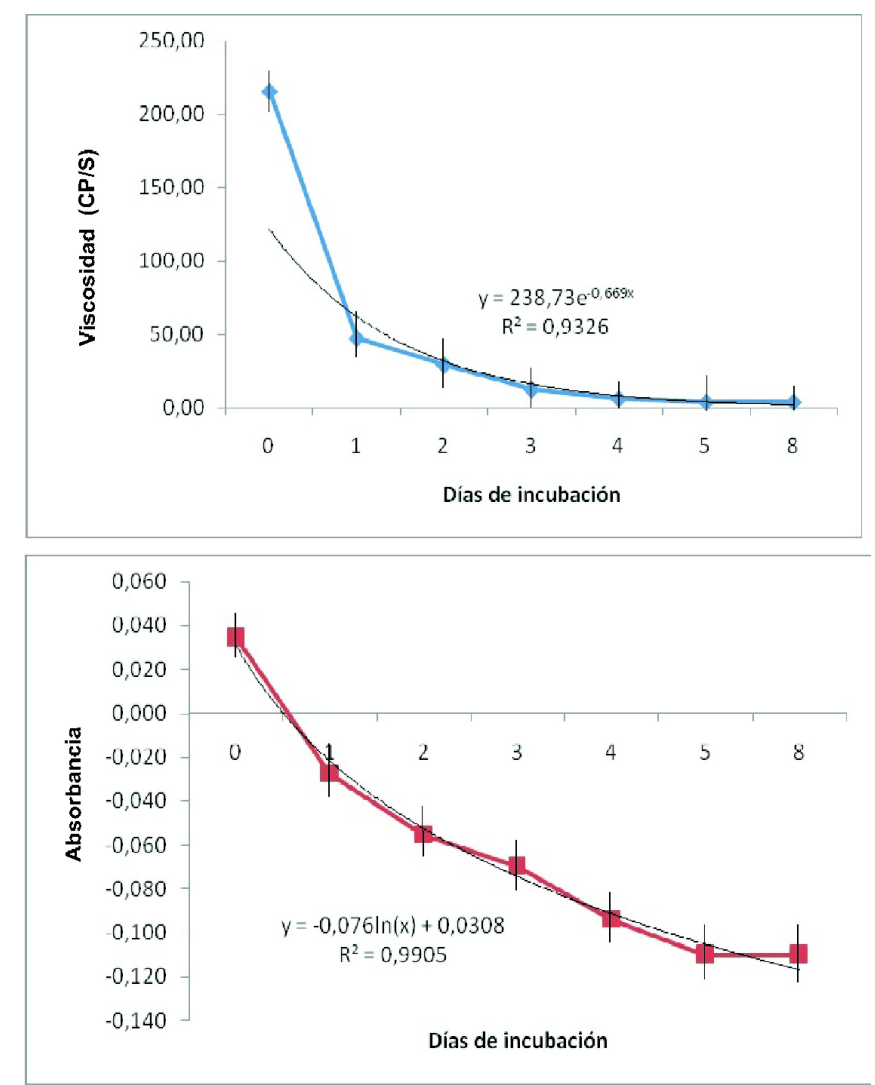

Figura 2. Comportamiento del medio de cultivo expuesto a T. viride en el tiempo, A) Viscosidad; B) Absorbancia

Luego de la incubación, la absorbancia cambió de comportamiento matemático de exponencial a logarítmico debido a la pérdida de coloración que genero $T$. viride en el medio de cultivo. La viscosidad mantuvo el comportamiento exponencial. Esto causó que no se pudiera establecer una relación lineal entre las variables con un coeficiente de determinación superior a 0,30. Por tanto, los datos de absorbancia fueron transformados adicionando el coeficiente 0,110 para nivelar la máxima pérdida de coloración con el cero. El análisis de regresión permitió establecer la siguiente ecuación: absorbancia transformada $=0,0603$ - 0,00922 Día $+0,000405$ Viscosidad cP/s con un coeficiente de determinación de 0,959 (Fig 3). La figura 3 muestra que el comportamiento logarítmico de los datos se mantuvo a pesar de la transformación de los datos. 


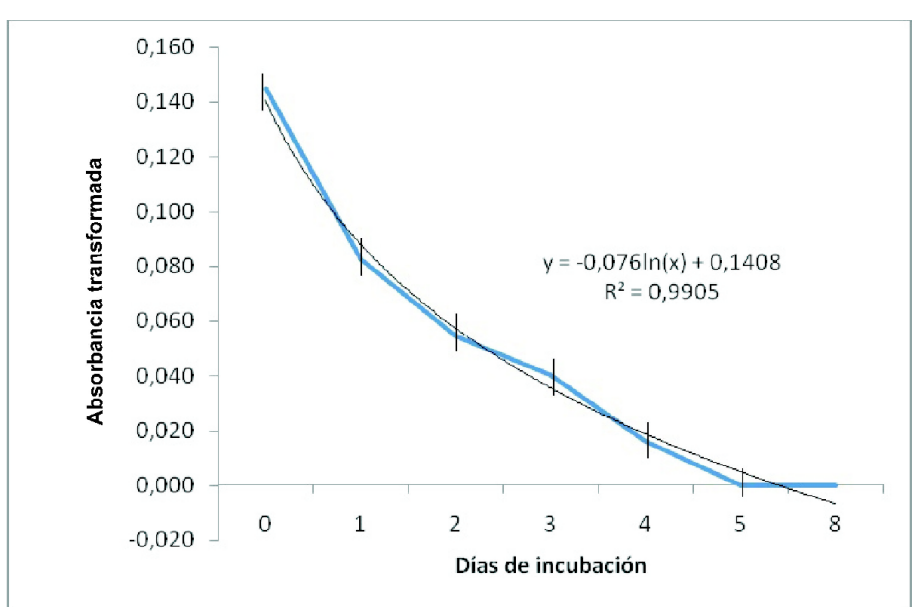

Figura 3. Relación entre la absorbancia transformada del medio de cultivo durante el crecimiento del $T$. viride

La técnica de DNS detectó que $T$. viride produce $0,066 \pm 0,001 \mathrm{micromoles} \mathrm{enzima/minL} \mathrm{es} \mathrm{decir,} \mathrm{que}$ en 5 días de reacción se producen $0,475 \pm 0,001$ micromoles de enzima/ml, correlacionando este dato con la pérdida de 0,075 unidades de absorbancia detectada, se encuentra que una unidad de pérdida de absorbancia equivale a $0,006 \pm 0,001 \mathrm{micromoles}$ de enzima/ml.

\section{Cepas problema}

Establecida la relación de unidades de enzima con la pérdida de absorbancia del medio, se evaluó tres cepas problema de diferente género fúngico y al control $T$. viride. La prueba de DNS indicó que la cepa 055C (Penicillium sp) produjo 0,035 $\pm 0,001 \mathrm{mi}-$ cromoles de enzima/minL, la cepa 019C (Trichoderma $s p$ ) produjo $0,133 \pm 0,001$ micromoles de enzima/minL y a la cepa 065C (Actinomucor $s p$ ) no se le detectó actividad endoglucanasa.

EI ANOVA para la viscosidad mostró que hubo una diferencia significativa entre el medio de cultivo CMC $y$ el efecto de los hongos con un valor $P=0,000$ $(\alpha=0,05)$. La prueba de Dunnett indicó que las cepas 019C, 055C y T. viride pertenecían al grupo de significanciaA y la cepa 065C junto con el blanco pertenecen al grupo $B$, es decir la viscosidad por sí misma, no permitió diferenciar todas las cepas (Fig 4A). En contraste, el ANOVA para absorbancia mostró que hubo una diferencia significativa entre los tratamientos con un valor $P=0,000$. La prueba de Dunnett mostró que hubo cuatro grupos de significancia el A: T. viride y 019C; B: 055C, C: 065C y el D: el blanco, es decir esta variable es más sensible (Fig 4B).

La cepa $065 \mathrm{C}$ tiene una actividad endoglucanasa 0,727 micromoles enzima/ml; la cepa 019C 0,929 micromoles enzima/ml; $T$. viride 1,065 micromoles enzima/ml y la cepa 019C 1,098 micromoles enzima/ml. Es decir el método permitió diferenciar claramente la actividad endoglucanasa de las distintas cepas y además se pudo cuantificar la degradación del medio de cultivo CMC-Rojo Congo con unidades comparables no arbitrarias. Este procedimiento es más sencillo que el DNS y es más sensible, ya que a la cepa $065 \mathrm{C}$ se le pudo detectar su actividad endoglucanasa. 


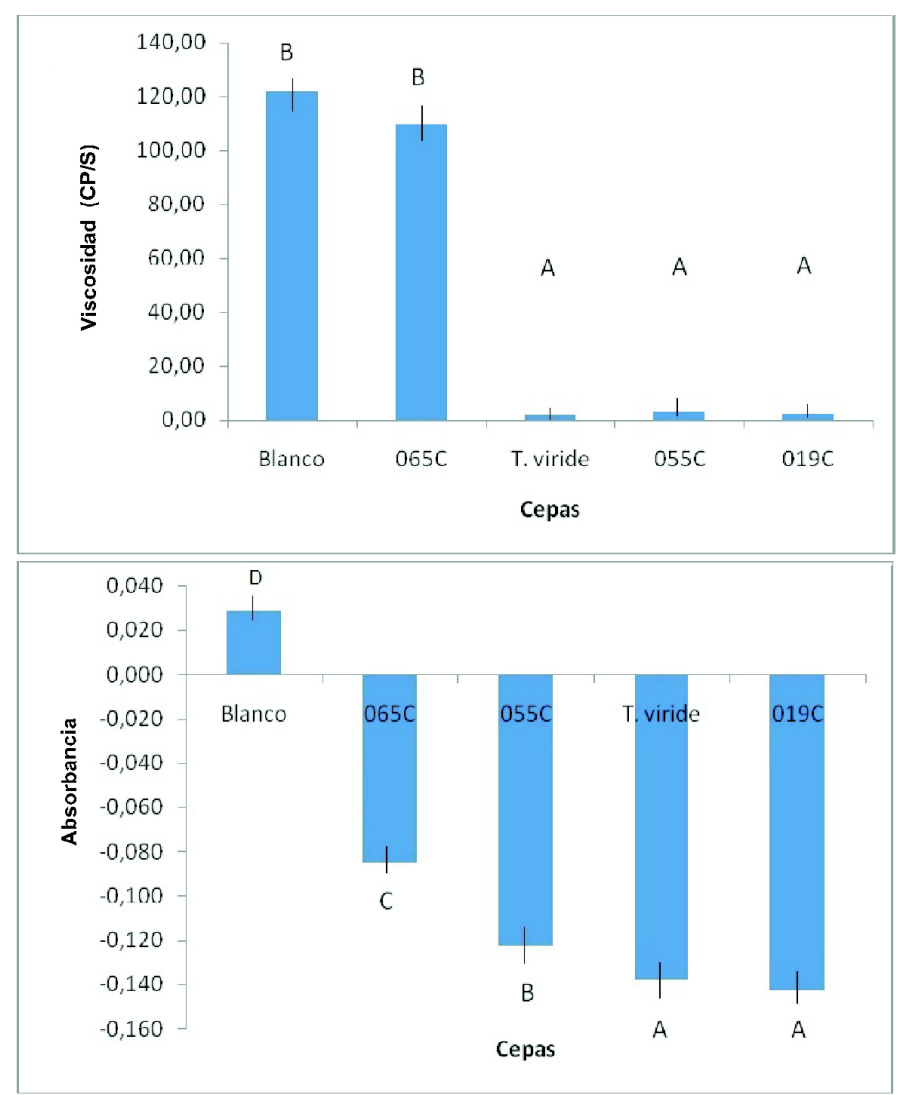

Figura 4. Comportamiento de las características del medio de cultivo CMC bajo el efecto de aislamientos fúngicos A) Viscosidad; B) Absorbancia

\section{DISCUSIÓN}

Sengupta et al. (2000) analizaron las limitaciones del método de referencia DNS para cuantificar azucares reductores utilizado para estimar la actividad enzimática glicosídica y encontraron que la lentitud y laboriosidad del método limita la capacidad de procesar un gran número de aislamientos y que de acuerdo a la enzima analizada se le deben incluir factores de corrección, ya que algunos azúcares productos pueden no ser detectados. Adicionalmente la sensibilidad 20-180 $\mathrm{mg} / \mathrm{ml}$ de glucosa, hace que muchas cepas aparezcan con una actividad enzimática no detectable, lo que implica tener que trabajar con una mayor cantidad de inóculo y volumen de muestra.
Chhabra \& Kelly (2002) utilizan DNS y simultáneamente Somogyi-Nelson para aumentar la sensibilidad en la cuantificación de azúcares reductores producto de endoglucanasas bacterianas, pero González \& Castellanos (2003) sugieren realizar modificaciones al método de Somogyi-Nelson dada la toxicidad de los reactantes que involucra y los riesgos para sus usuarios.

Cuando no existen limitaciones en la cantidad de muestra como en procesos de fermentación, el método DNS es ampliamente usado (Hong et al. 2001; Srinivasan et al. 200; Nomachi et al. 2002), 
pero en procesos de screening con múltiples aislamientos los grandes volúmenes de reacción son imprácticos.

El medio de cultivo con CMC y rojo Congo tiene una absorbancia que es función de la viscosidad y la concentración de CMC (con un coeficiente de determinación del 0,996), es decir a mayor concentración de CMC se forma una mayor cantidad de complejo CMC-rojo Congo. Esta capacidad de unirse a polisacáridos ha sido utilizada como herramienta diagnóstica en clínica y en otras aplicaciones (Pire 1985; Berkhoff \& Vinal 1986; Guzman et al. 1999; Hernández et al. 2000; Bély et al. 2006). Este medio de cultivo CMC-rojo Congo al ser expuesto a un hongo celulolítico rápidamente pierde viscosidad y absorbancia, debido a que las endoglucanasas rompen la estructura amorfa de la CMC (Ohmiya et al. 2003; Aro et al. 2005). En el caso de T. viride se generó una pérdida de viscosidad del $98,2 \%$ y una disminución de la absorbancia del $300 \%$ en 5 días. Es decir, que en un $25 \%$ del tiempo empleado

\section{CONCLUSIÓN}

La medición de la pérdida de absorbancia del medio de cultivo CMC-rojo Congo mostró ser una vía rápida y efectiva para cuantificar la actividad

\section{AGRADECIMIENTOS}

$\mathrm{Al}$ apoyo técnico prestado por el personal del Instituto de Ciencia y Tecnología (ICTA) y al

\section{REFERENCIAS}

Asociation of Analytical Communities-AOAC. 2000. Oficial methods of analysis. AOAC International. William Horwitz, Ed. Washington D.C.

Aro N, Pakula T, Penttilä M. Transcriptional regulation of plant cell wall degradation by filamentous fungi. FEMS Microbiology Reviews 2005; 29:719739.

Bély M, Makovitzky J. Sensitivity and specificity of red Congo staining according to Romhányi, com- en la realización de la prueba DNS, el medio CMCrojo Congo mostró cambios considerables en la absorbancia y la viscosidad, que fueron utilizados para estimar la actividad enzimática fúngica.

Al evaluar el efecto de diferentes cepas de hongos sobre el medio CMC-rojo Congo, el análisis de varianza mostró que la pérdida de absorbancia del medio CMC-rojo Congo discriminó la actividad enzimática de las cepas, a diferencia de la viscosidad. Es decir que las alteraciones en la absorbancia del medio CMC-rojo Congo se pueden correlacionar directamente con la acción del aparato enzimático de los hongos celulolíticos.

En el presente trabajo se evaluó una cepa de Actinomucor sp (065C) que con el método de DNS no se le detectó actividad enzimática y que con el medio CMC-rojo Congo, no sólo se le pudo detectar actividad enzimática sino que fue cuantificada en 0,727 micromoles enzima/ml. Por tanto, el método basado en el complejo CMC-rojo Congo superó en sensibilidad al método de referencia DNS.

enzimática endoglucanasa en hongos, con una clara coherencia experimental y sensibilidad superior a la del método DNS.

Instituto de Biotecnología (IBUN) de la Universidad Nacional de Colombia.

parison with Puchtler's or Benhold's methods. Acta histochemica 2006; 108:175-180.

Berkhoff H, Vinal C. Congo red medium to distinguish between invasive and non-invasive Escherichia coli pathogenic for poultry. Avian diseases 1986; 30 (1):117-121.

Brasil P, Freer J, Siika-Aho M, Ferraz A. Extraction and determination of enzymes produced by Ceriporiopsis subvermispora during biopulping of 
Pinus taeda wood chips. Enzyme and microbial technology 2004; 34:228-234.

Chhabra S, Kelly R. Biochemical characterization of Thermotoga maritima endoglucanase Cel74 with and without a carbohydrate binding module (CBM). FEBS Letters 2002; 531: 375-380.

Contreras-Pinzón M, Dominguez-Espinosa R, González-BurgosA. Proceso de biotransformación láctica del jugo de Aloe vera. Tecnología, ciencia, educación 2007; 22(1):35-42.

González G, Castellanos O. Alternativas de modificación del método de Somogyi-Nelson para la determinación de azúcares reductores a partir de sus posibilidades químicas. Revista Ingeniería e Investigación 2003; 52: 5-17.

Guzmán G, Ramírez F, Tapia F, Navarro P. Las especies del género Psilocybe (Fungi, Basidiomycotina, Agaricales) conocidas de Veracruz (México). Acta Botánica Mexicana 1999; 49:35-46.

Hernández A, Garcia D, Soroa M, HernándezA. Estudio de algunos géneros bacterianos asociados a la rizósfera de los cultivos de Gerbera (Gerbera jamesonii) y clavel (Dianthus barbatus, Dianthus caryophyllus). Cultivos tropicales. 2000; 21(3):15-18.

Hong J, Tamaki H, Akiba S, Yamamoto K, Kumagai $\mathrm{H}$. Cloning of a gene enconding a highly stable endo $\beta 1$,4glucanase on cellulose biosynthesis in Acetobacter xylinum ATCC23769. Journal of bioscience and bioengineering 2001; 92 (5):434-441.

International Union of Pure and Applied Chemistry-IUPAC 1984. Commision on Biotechnology, in "Measurement of cellulase activities" (T.K. Ghose, ed.). Biochemical Engineering Research Centre, Indian institute of Technology. New Delhi.
Nomachi W, Urago K, Oka T, Ekino K, Matsuda M, Goto M, Furukawa K. Molecular breeding of Aspergillus kawachii overproducing cellulase and its application to brewing barley Shochu. Journal of bioscience and bioengineering 2002; 93(4): 382-387.

Ohmiya K, Sakka, K, Kimura, T, Morimoto K. Application of microbial genes to recalcitrant biomass utilization and environmental conservation, Journal of Bioscience and Bioengineering 2003; 95(6): 549-561.

Pire R. Densidad longitudinal de raíces y extracción de humedad en un viñedo de el Tocuyo Venezuela. Agronomía Tropical 1985; 35(1-3):5-20.

Rautela G, Cowling E. Simple cultural test for relative cellulolytic activity of fungi. Applied and environmental microbiology. 1966; 14:892.

Sengupta S, Jana M, Sengupta D, Naskar A. A note on the estimation of microbial glycosidase activities by dinitrosalicylic acid reagent. Applied microbiology and biotechnology 2000; 53: 732-735.

Smith R. Rapid tube test for detecting fungal cellulase production. Applied and environmental microbiology. 1977; 33: 980.

Srinivasan K, Murakami M, Nakashimada Y, Nishio N. Efficient production of cellulolytic and xylanolytic enzymes by the rumen anaerobic fungus, Neocallimastix frontalis in a repeated batch cultura. Journal of bioscience and bioengineering 2001; 91(2):153-158.

Teather R, Wood P. Use of red Congo-polysaccharide interactions in enumeration and characterization of cellulolytic bacteria from the bovine rumen. Applied and environmental microbiology 1982; 43(4):777-780.

Thorn G, Reddy A, Harris D, Paul E. Isolation of saprophytic basidiomycetes from soil. Applied and environmental microbiology 1996; 62(11):4288-4292.

Wood T, Bhat K. 1988. Methods for measuring cellulase activities, in Measuring cellulose activities. Academic Press Inc. New York. 